\title{
Influence of Fractal Kinetics on Molecular Recognition
}

\author{
Michael A. Savageau \\ Department of Microbiology and Immunology, The University of Michigan Medical School, Ann Arbor, MI 48109-0620, \\ USA
}

\begin{abstract}
Molecular recognition is a central issue for nearly every biological mechanism. The analysis of molecular recognition to date has been conducted within the framework of classical chemical kinetics, in which the kinetic orders of a reaction have positive integer values. However, recent theoretical and experimental advances have shown that the assumptions inherent in this classical framework are invalid under a variety of conditions in which the reaction environment may be considered nonideal. A good example is provided by reactions that are spatially constrained and diffusion limited. Bimolecular reactions confined within two-dimensional membranes, one-dimensional channels or fractal surfaces in general exhibit kinetic orders that are noninteger. An appropriate framework for the study of these nonideal phenomena is provided by the Power-Law formalism, which includes as special cases the Mass-Action formalism of chemical kinetics and the Michaelis-Menten formalism of enzyme kinetics. The Power-Law formalism is an appropriate representation not only for fractal kinetics per se, but also for other nonideal kinetic phenomena, provided the range of variation in concentration is not too large. After defining some elementary concepts of molecular recognition, and showing how these are manifested in classical kinetic terms, this paper contrasts the implications of classical and fractal kinetics in a few simple cases. The principal distinction lies in the ability of fractal kinetics to nonlinearly transform, rather than proportionally transmit, the input $S / N$ ratio. As a consequence, fractal kinetics create a threshold for the input signal below which no recognition occurs and above which amplified recognition takes place. Thus, fractal kinetics implies an intimate relationship between design of the physiological mechanisms regulating the environment of the process and design of the molecular process itself. These results also suggest that recognition in the presence of a favorable input ratio would emphasize rapid reactions, while recognition in the presence of an unfavorable input ratio would emphasize slow reactions.
\end{abstract}

\section{INTRODUCTION}

It has long been appreciated that molecular recognition is central to nearly every biological mechanism, whether one is concerned with ligand binding to cell surface receptors in hormone action or antigen presentation, protein-protein interaction in intracellular signaling, or the modulation of transcription in the nucleus by transactivating proteins targeted to specific DNA sequences. Hence, the study of molecular recognition has conceptual importance for our understanding of the biological world; it also has practical importance for guiding our use of the new biotechnologies.

The analysis of molecular recognition to date has typically been conducted within the framework of classical chemical kinetics. Within this framework the kinetic orders of a reaction have positive integer values equal to the number of molecules of a given type that enter into the reaction (Erdi and Toth, 1989). However, reactions that occur within a nonideal environment, e.g., spatially restricted and diffusion limited reactions (Galfi and Racz, 1988; Jiang and Ebner, 1990; Koo and Kopelman, 1991), do not conform to the assumptions inherent in the classical framework. Recent studies have shown that elementary reactions confined to two-dimensional membranes, onedimensional channels, or fractal surfaces, as is typical of biological systems, exhibit kinetic orders that are noninteger (Kopelman, 1986; Newhouse and Kopelman, 1988). An alternative to the classical framework is necessary for the analysis of these phenomena.

The appropriate framework for dealing with fractal kinetics, and other nonideal kinetic phenomena involving noninteger kinetic orders, is provided by the Power-Law formalism (Savageau, 1993). The Power-Law formalism includes as a special case the Mass-Action formalism of chemical kinetics, which assumes no spatial restrictions. The Mass-Action formalism in turn includes as a special case the Michaelis-Menten formalism of enzyme kinetics, which assumes quasi-steady state for the enzyme forms and the absence of interactions among these forms. There can be no conflict among these formalisms when they are applied to phenomena that validly fit within the range of the more restrictive case. However, the most general of these formalisms clearly must be used to explore the implications of fractal kinetics for molecular recognition.

My goal in this paper is to describe some implications of fractal kinetics for molecular recognition in a few simple biological processes. However, I shall first define elementary aspects of molecular recognition and show how these are manifested in classical kinetic terms. I shall then come to the main point and contrast the classical and fractal implications for molecular recognition. Finally, I shall conclude with a brief discussion of molecular recognition and speculate on the relevance of fractal kinetics in signal transduction. 


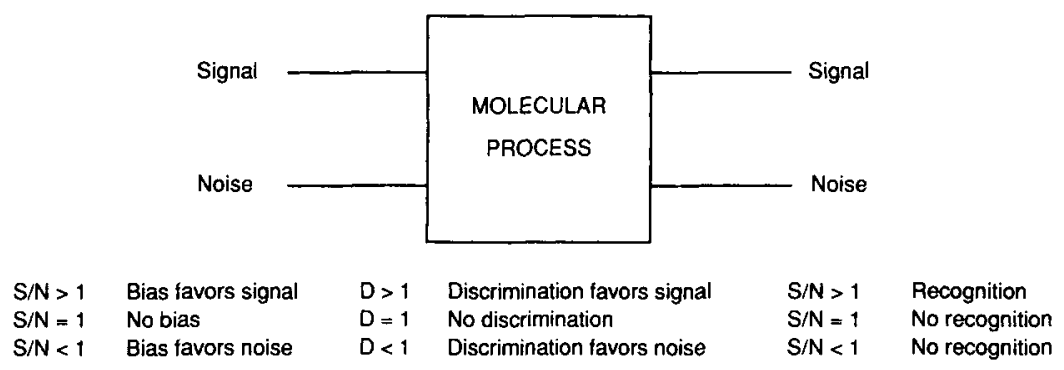

Figure 1. Conceptual framework for analysis of molecular recognition. See text for discussion.

\section{ELEMENTARY CONCEPTS}

I shall consider the recognition process to consist of three aspects: presentation, transformation, and recognition. The simplest case is depicted in Fig. 1. Presentation occurs within an environment offering two structurally related molecular species. One is the species of interest, and its abundance or concentration is considered the signal in the input being presented. The abundance or concentration of the alternative species is considered the noise in the input being presented. Transformation of the inputs is accomplished by a molecular process that discriminates among the alternatives presented and in so doing generates a related set of outputs. Recognition is manifested in the ratio of output signal to output noise, which is considered the degree of recognition achieved by this process. Let us amplify these notions a bit and then give some concrete examples.

The input being presented by the environment has an associated property we shall call the bias, which is defined as the $\mathrm{S} / \mathrm{N}$ ratio in the input. A ratio of one implies no bias; a ratio greater than one implies bias in favor of the signal; a ratio less than one implies bias in favor of the noise.

The transformation carried out by the molecular process can be either a simple discrimination or a composite discrimination, which in turn might be dissected into a number of simple discrimination steps. The discrimination factor is a measure of the relative specificity of the molecular process for the input molecules.

As noted above, recognition is defined in terms of the output $\mathrm{S} / \mathrm{N}$ ratio. The greater the ratio, the greater the degree of recognition. In a qualitative sense, ratios greater than one can be considered recognition, whereas ratios less than or equal to one can be considered no recognition. The outputs generated may be either concentrations, in which case recognition is measured by the extents of reactions, or fluxes, in which case recognition is measured by the rates of reactions.

\section{Recognition by simple kinetic discrimination}

Consider a simple elementary reaction presented with two alternative substrates as depicted in Fig. 2. The concentrations $X_{1}$ and $X_{2}$ will be considered the input signal and noise, respectively. Hence, the bias is given by the ratio $X_{1} / X_{2}$. The strengths with which these input molecules are acted upon by the elementary molecular process are the rate constants $\alpha_{3}$ and $\alpha_{4}$. The discrimination ratio $D$ is given by their ratio $\alpha_{3} / \alpha_{4}$. The ratio of output fluxes provides the measure of recognition in this process. Hence:

$$
R=\frac{v_{3}}{v_{4}}=\frac{\alpha_{3} X_{1}}{\alpha_{4} X_{2}}=D \frac{X_{1}}{X_{2}}
$$

If there is an unbiased input $\left(X_{1}=X_{2}\right)$ and no discrimination $(D=1)$, then no recognition can take place $(R=1)$. In order for recognition to occur $(R>1)$, either the input presented must be biased in favor of the signal $\left(X_{1}>X_{2}\right)$ when there is no discrimination, or there must be discrimination in favor of the signal $(D>1)$ when there is no bias, or both.

\section{Recognition by Composite Kinetic Discrimination}

A composite molecular process involving a number of elementary discrimination steps is shown in Fig. 3. If in the reverse step there is discrimination in favor of the noise $\left(\beta_{4}>\beta_{3}\right)$, then the reverse steps provide an elementary form of noise suppression or proofreading.

Again, the concentrations $X_{1}$ and $X_{2}$ will be considered the input signal and noise, and the input bias is given by the ratio $X_{1} / X_{2}$. The first discrimination step in the forward direction has a discrimination factor $\alpha_{3} / \alpha_{4}$, the second discrimination step in the forward direction (with the intermediates $X_{3}$ and $X_{4}$ as inputs) has a discrimination factor $\alpha_{5} / \alpha_{6}$, and the discrimination step in the reverse direction (again with intermediates $X_{3}$ and $X_{4}$ as inputs) has a discrimination factor $\beta_{3} / \beta_{4}$. What constitutes recognition in this composite process?

In steady state one can carry out the straightforward calculations and show that $R$ is given by the following expression:

$$
\begin{aligned}
& R=\frac{v_{5}}{v_{6}}=\left[\frac{\alpha_{3} \alpha_{5}\left(\alpha_{6}+\beta_{4}\right)}{\alpha_{4} \alpha_{6}\left(\alpha_{5}+\beta_{3}\right)}\right] \frac{X_{1}}{X_{2}}=D \frac{X_{1}}{X_{2}}
\end{aligned}
$$

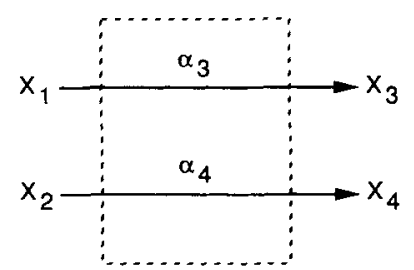

Figure 2. Simple kinetic discrimination. The proper substrate $X_{1}$ and an analog substrate $X_{2}$ are transformed by an elementary chemical reaction into the corresponding products $X_{3}$ and $X_{4}$. The rate constants associated with these transformations are $\alpha_{3}$ and $\alpha_{4}$, respectively. 


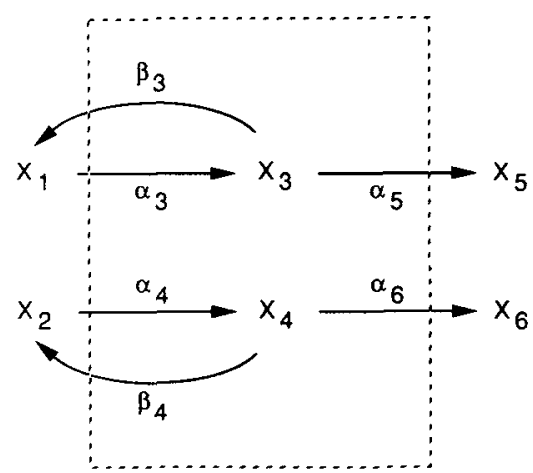

Figure 3. Composite kinetic discrimination. There are two simple kinetic discrimination steps in the forward direction and one in the reverse direction. The first characterizes the transformation of substrates into intermediates (this is identical to that in Fig. 2), the second characterizes the transformation of intermediates into products, and the third characterizes the transformation of intermediates back to substrates.

There are two extreme cases that easily can be considered. If $\beta_{3} \ll \alpha_{5}$ and $\beta_{4} \ll \alpha_{6}$, then:

$$
R=\left(\frac{\alpha_{3}}{\alpha_{4}}\right) \frac{X_{1}}{X_{2}}=D_{1} \frac{X_{1}}{X_{2}}=D \frac{X_{1}}{X_{2}}
$$

In this case the noise suppression step is negligible and the discrimination achieved is equal to that of the first step alone. Functionally, this is no different than the case in the previous subsection. On the other hand, if $\beta_{3} \gg \alpha_{5}$ and $\beta_{4} \gg \alpha_{6}$, then:

$$
R=\left(\frac{\alpha_{3}}{\alpha_{4}}\right)\left(\frac{\alpha_{5}}{\alpha_{6}}\right)\left(\frac{\beta_{4}}{\beta_{3}}\right) \frac{X_{1}}{X_{2}}=\left(\frac{D_{1} D_{2}}{D_{3}}\right) \frac{X_{1}}{X_{2}}=D \frac{X_{1}}{X_{2}}
$$

Recognition in this case can be greatly enhanced over that possible with a single elementary step. If the discrimination in each of the forward steps favors the signal and in the reverse step favors the noise, then the overall discrimination becomes in effect the product of three factors each of which is greater than one.

\section{CLASSICAL EXAMPLES}

Two of the most common types of recognition that have been treated within the classical framework involve equilibrium binding for alternative ligands of a receptor and steady-state reaction rates for alternative substrates of an enzyme.

\section{Recognition by receptor equilibrium discrimination}

The ability of a cell surface receptor to recognize a specific ligand in the presence of a structurally related alternative currently is of considerable interest because it represents the first stage of signal transduction (Brugge, 1993; Marx, 1993). A simplified representation of this process is given in Fig. 4. The input signal molecule $X_{1}$ competes with the alternative $X_{2}$ for binding to the free receptor $X_{s}$. Recognition is measured by the output ratio of signal to noise bound to receptor $X_{3} / X_{4}$ at equilibrium.

Transformation by the molecular process is governed by the following equations:

$$
\mathrm{d} X_{3} / \mathrm{d} t=\alpha_{3} X_{1} X_{5}-\beta_{3} X_{3}
$$

$$
\begin{gathered}
\mathrm{d} X_{4} / \mathrm{d} t=\alpha_{4} X_{2} X_{5}-\beta_{4} X_{4} \\
X_{5}=X_{\mathrm{T}}-X_{3}-X_{4}
\end{gathered}
$$

where $X_{\mathrm{T}}$ is the concentration of total receptor, including that which is unbound $X_{5}$, that which is bound with signal $X_{3}$, and that which is bound with noise $X_{4}$. At equilibrium:

$$
\begin{aligned}
& X_{3}=\frac{\alpha_{3}}{\beta_{3}} X_{1} X_{5} \\
& X_{4}=\frac{\alpha_{4}}{\beta_{4}} X_{2} X_{5}
\end{aligned}
$$

and the degree of recognition is then:

$$
R=\frac{X_{3}}{X_{4}}=\left(\frac{\alpha_{3}}{\alpha_{4}}\right)\left(\frac{\beta_{4}}{\beta_{3}}\right) \frac{X_{1}}{X_{2}}=\left(\frac{D_{1}}{D_{2}}\right) \frac{X_{1}}{X_{2}}=D \frac{X_{1}}{X_{2}}
$$

Note that the outputs in the previous examples were fluxes, whereas the outputs in this case are concentrations.

There are two elemental discriminations involved here. If association discriminates in favor of the input signal $\left(D_{1}>1\right)$ and dissociation discriminates in favor of the output noise $\left(D_{2}<1\right)$, then the overall discrimination can be greater than would be possible by simple kinetic discrimination. In this simple case, it is clear that overall discrimination also is equal to the ratio of equilibrium constants, i.e.:

$$
D=\frac{K_{e 1}}{K_{e 2}}
$$

One could have solved Eqns. (5)-(7) for the equilibrium values of $X_{3}$ and $X_{4}$ and then taken their ratio. This procedure is much more involved, but in the end one would obtain exactly the same results. The simpler procedure works because it is not really necessary to know the values of $X_{3}, X_{4}$, and $X_{5}$. It is enough to know that the value of $X_{5}$ is immaterial because it cancels out when the ratio is taken.

\section{Recognition by enzyme kinetic discrimination}

The specificity of enzymes for their substrates is not absolute (Fersht, 1985). There always will be substrate analogues in any complex environment, which raises

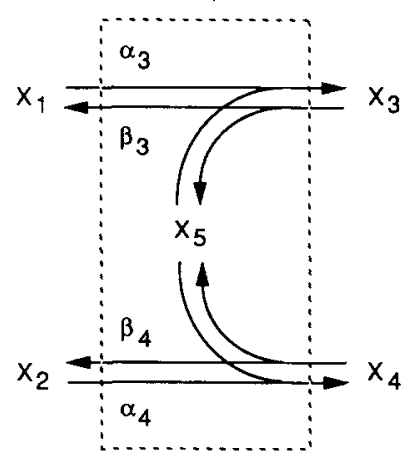

Figure 4. Receptor equilibrium discrimination. The proper ligand $X_{1}$ and its analog $X_{2}$ associate with the unbound receptor $X_{5}$ to yieid the proper complex of receptor-ligand $X_{3}$ and the analog complex of receptor-ligand $X_{4}$. The complexes also dissociate to yield ligand and receptor in their unbound forms. The rates of association and dissociation are identical at equilibrium. 


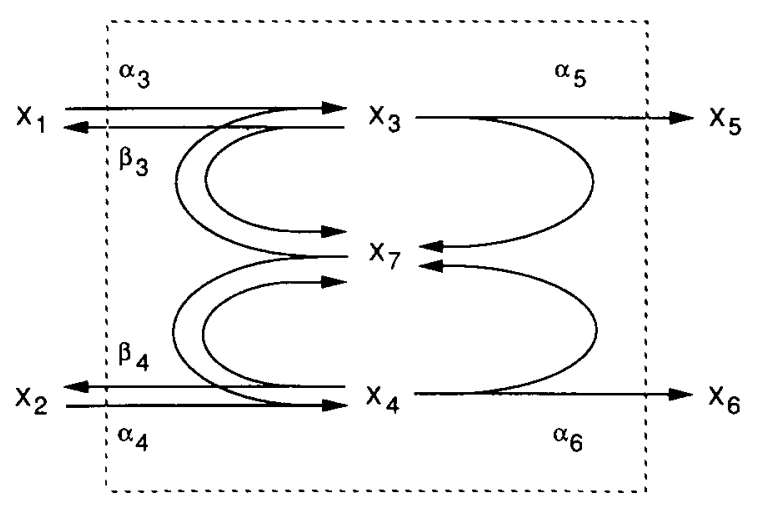

Figure 5. Enzyme kinetic discrimination. The proper substrate $X_{1}$ and the analog substrate $X_{2}$ associate with the free form of the enzyme $X_{7}$ to yield enzyme-substrate complexes $X_{3}$ and $X_{4}$. respectively. The complexes in turn dissociate to yield free enzyme and either the original substrates or the corresponding products $X_{5}$ and $X_{6}$. Both the top half and the bottom half of this diagram represent conventional Michaelis-Menten mechanisms that are assumed to operate in a quasi-steady state far from equilibrium.

the question of an enzyme's ability to recognize its proper substrate. The classical Michaelis-Menten representation of this process is shown in Fig. 5. The input signal is again represented by the concentration of the proper substrate $X_{1}$, and the input noise is represented by the concentration of the analogue $X_{2}$; they compete for binding to the free enzyme $X_{7}$. The enzymereactant complexes are represented by $X_{3}$ and $X_{4}$; in each case, they can either dissociate or undergo reaction to yield free enzyme and the relevant product. The output in this case is taken to be $v_{5}$, the flux of the proper product $X_{5}$, and $v_{6}$, the flux of the analog product $\boldsymbol{X}_{6}$.

Transformation by the molecular process is governed by the following equations:

$$
\begin{gathered}
\mathrm{d} X_{3} / \mathrm{d} t=\alpha_{3} X_{1} X_{7}-\left(\alpha_{5}+\beta_{3}\right) X_{3} \\
\mathrm{~d} X_{4} / \mathrm{d} t=\alpha_{4} X_{2} X_{7}-\left(\alpha_{6}+\beta_{4}\right) X_{4} \\
X_{7}=X_{T}-X_{3}-X_{4}
\end{gathered}
$$

where $X_{\mathrm{T}}$ is the concentration of total enzyme including that which is free $X_{7}$, that which is complexed with the proper substrate $X_{3}$, and that which is complexed with the analog substrate $X_{4}$. At steady state $\left(\mathrm{d} X_{i} / \mathrm{d} t=0\right)$ with negligible product concentration:

$$
\begin{aligned}
& X_{3}=\frac{\alpha_{3}}{\alpha_{5}+\beta_{3}} X_{1} X_{7} \\
& X_{4}=\frac{\alpha_{4}}{\alpha_{6}+\beta_{4}} X_{2} X_{7}
\end{aligned}
$$

Recognition is then measured by the ratio:

$$
R=\frac{v_{5}}{v_{6}}=\frac{\alpha_{5} X_{3}}{\alpha_{6} X_{4}}=\left[\frac{\alpha_{3} \alpha_{5}\left(\alpha_{6}+\beta_{4}\right)}{\alpha_{4} \alpha_{6}\left(\alpha_{5}+\beta_{3}\right)}\right] \frac{X_{1}}{X_{2}}=D \frac{X_{1}}{X_{2}}
$$

There are two extreme cases that easily can be considered. If dissociation is very slow in each case, $\beta_{3} \ll \alpha_{5}$ and $\beta_{4} \ll \alpha_{6}$, then:

$$
R=\left(\frac{\alpha_{3}}{\alpha_{4}}\right) \frac{X_{1}}{X_{2}}=D_{1} \frac{X_{1}}{X_{2}}=D \frac{X_{1}}{X_{2}}
$$

In this case the noise suppression step is negligible and the discrimination achieved is equal to that of the first step alone. Functionally, this is no different than the case discussed earlier. On the other hand, if dissociation is very fast in each case, $\beta_{3} \gg \alpha_{5}$ and $\beta_{4} \gg \alpha_{6}$, then:

$$
R=\left(\frac{\alpha_{3}}{\alpha_{4}}\right)\left(\frac{\alpha_{5}}{\alpha_{6}}\right)\left(\frac{\beta_{4}}{\beta_{3}}\right) \frac{X_{1}}{X_{2}}=\left(\frac{D_{1} D_{2}}{D_{3}}\right) \frac{X_{1}}{X_{2}}=D \frac{X_{1}}{X_{2}}
$$

Recognition in this case can be greatly enhanced over that possible with a single elementary step. If the discrimination in each of the forward steps favors the signal and in the reverse step favors the noise, then the overall discrimination becomes in effect the product of three factors each of which is greater than one. It should be noted that values of the $\alpha$ and $\beta$ rate constants are constrained by the values of the overall equilibrium constants and the values of $\beta_{5}$ and $\beta_{6}$, the rate constants for the binding of product molecules, which are being neglected here because product concentrations are assumed to be negligible.

In this simple case, it is clear that overall discrimination also is equal to $k_{\text {cat1 }} / K_{\mathrm{m} 1}$ divided by $k_{\mathrm{cat} 2} / K_{\mathrm{m} 2}$ (Fersht, 1985), i.e.:

$$
D=\frac{k_{\text {cat } 1} / K_{\mathrm{m} 1}}{k_{\text {cat } 2} / K_{\mathrm{m} 2}}
$$

It is important to note that this system is operating far from thermodynamic equilibrium and that recognition is based on kinetic discrimination. If the reactions were allowed to reach equilibrium, the ratio of output concentrations then would be the more appropriate measure of recognition. However, in this instance, the kinetic properties of the enzyme become irrelevant, and discrimination is given by the ratio of equilibrium constants.

In analogy with the previous example, one could have solved Eqns. (12)-(14) for the steady-state values of $X_{5}$ and $X_{6}$ and then taken the appropriate ratio. This procedure is much more involved, but in the end one would obtain exactly the same results. Again, the simpler procedure works because it is not really necessary to know the values of $X_{5}, X_{6}$ and $X_{7}$. It is enough to know that the value of $X_{7}$ is immaterial because it cancels out when the ratio is taken.

\section{Graphical representation of molecular recognition}

In the examples of the previous subsections, the equation for the overall recognition process has exactly the same form, i.e.:

$$
R=D \frac{X_{1}}{X_{2}}
$$

Consequently, overall recognition as a function of input bias has a particularly simple plot in a logarithmic coordinate system (Fig. 6). The reasons for this choice of plot will become clear in the following section.

\section{FRACTAL IMPLICATIONS}

The rate of a reaction involving the collision of a pair of molecules is proportional to the joint probability of their being at the same place at the same time. To a first 
approximation, in a homogeneous three-dimensional space, this probability is given by the product of their concentrations. This is the basis for the Mass-Action formalism. However, when such collisions occur within a spatially restricted region, the rate of reaction involves concentrations raised to noninteger powers (Kopelman, 1986; Newhouse and Kopelman, 1988). These powers alter the effect of input bias on the overall recognition process. This is perhaps best appreciated in the context of familiar examples.

\section{Recognition by receptor equilibrium discrimination}

Let us revisit the analysis of ligand recognition by cell surface receptors and assume that the molecular processes in Fig. 4 occur within a two-dimensional membrane or a one-dimensional channel. Recall that recognition is measured at equilibrium by the ratio of receptor bound with output signal to that bound with output noise $X_{3} / X_{4}$.

The transformation process is now governed by the following equations in the Power-Law formalism:

$$
\begin{aligned}
\mathrm{d} X_{3} / \mathrm{d} t & =\alpha_{3} X_{1}^{g_{31}} X_{5}^{g_{35}}-b_{3} X_{3} \\
\mathrm{~d} X_{4} / \mathrm{d} t & =\alpha_{4} X_{2}^{g_{42}} X_{5}^{g_{45}}-\beta_{4} X_{4} \\
X_{5} & =X_{\mathrm{T}}-X_{3}-X_{4}
\end{aligned}
$$

At equilibrium:

$$
\begin{aligned}
& X_{3}=\frac{\alpha_{3}}{\beta_{3}} X_{1}^{g_{31}} X_{5}^{g_{35}} \\
& X_{4}=\frac{\alpha_{4}}{\beta_{4}} X_{2}^{g_{42}} X_{5}^{g_{45}}
\end{aligned}
$$

and the degree of fractal recognition, which will be denoted by the symbol $R_{f}$, is then:

$$
R_{\mathrm{f}}=\frac{X_{3}}{X_{4}}=\left(\frac{\alpha_{3}}{\alpha_{4}}\right)\left(\frac{\beta_{4}}{\beta_{3}}\right) \frac{X_{1}^{g_{31}} X_{5}^{g_{35}}}{X_{2}^{g_{42}} X_{5}^{g_{45}}}
$$

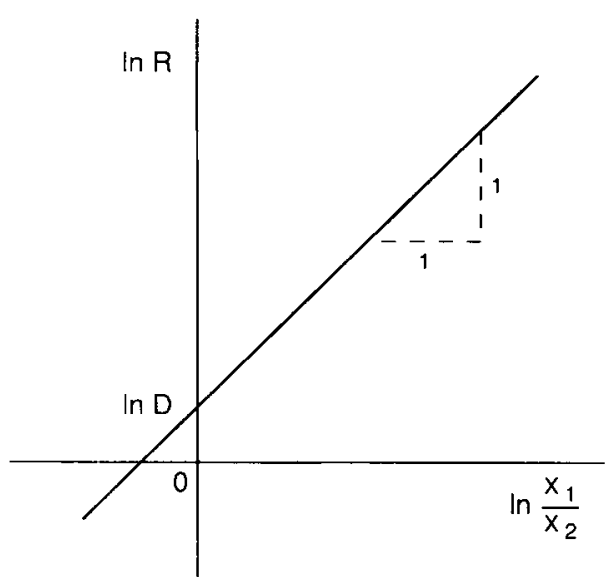

Figure 6. Degree of recognition as a function of the input bias for a classical recognition process. The logarithm of the input $\mathrm{S} / \mathrm{N}$ ratio (bias) is represented on the horizontal axis, and the logarithm of the output $S / N$ ratio (degree of recognition) is represented on the vertical axis. The molecular recognition function is a straight line with slope equal to unity. The intercept on the vertical axis gives the logarithm of the overall discrimination by the molecular process, which is equal to the degree of recognition with an unbiased input.
It is clear that the $X_{5}$ terms no longer cancel and that recognition is not a simple function of input bias. Nevertheless, one can determine the fractal recognition function numerically for any combination of values for the kinetic orders, rate constants, and total concentration of receptor. We will not pursue the general case here.

There are cases in which all the kinetic orders, to a good first approximation, are equal, and the analysis is greatly simplified. Galfi and Racz (1988) showed that the rate constant for a bimolecular reaction under diffusion-limited conditions scaled as a power function in time. They assumed for simplicity that the two substrates were identical (i.e., $\mathrm{A}+\mathrm{A} \rightarrow \mathrm{C}$ ), but also indicated that their results were more general. Jiang and Ebner (1990) showed explicitly that such scaling is true when the two substrates are different (i.e., $A+B \rightarrow C$ ), have different concentrations, and have different diffusion coefficients. As can be seen from their plotted results, the details of the spatial pattern of reaction do change with these parameters, but the scaling of the rate constant is unchanged. One readily can show that a conventional rate law with a rate constant that scales as a power function in time is equivalent to a time-invariant rate law that is a power function in the appropriate concentrations, and that the values of the individual kinetic orders in the case with $A \neq B$ are simply one-half the value of the kinetic order in the case with $A=B$ (Savageau, unpublished data). Under these conditions we can let $g_{31}=g_{42}=g_{35}=g_{45}=$ $g$, and the $X_{5}$ terms in the fractal recognition function [Eqn. (27)] now cancel as in the classical example. The simplified fractal recognition function then becomes:

$$
R_{\mathrm{f}}=\frac{X_{3}}{X_{4}}=\left(\frac{\alpha_{3}}{\alpha_{4}}\right)\left(\frac{\beta_{4}}{\beta_{3}}\right)\left(\frac{X_{1}}{X_{2}}\right)^{g}
$$

or

$$
R_{\mathrm{f}}=D\left(\frac{X_{1}}{X_{2}}\right)^{g}
$$

where $D$ is the overall discrimination factor as defined for the classical example.

It should be noted that the value of $D$ will undoubtedly change with spatial restriction, since the concentration equilibrium constant has been shown to increase under these conditions (Minton, 1992). However, we shall not be concerned with this thermodynamic effect here.

This fractal recognition function is plotted in Fig. 7 with values of $g$ that are associated with different degrees of spatial restriction. The homogeneous threedimensional case $(g=1)$ corresponds to the classical example. The progressively higher values of $g$ correspond to the two- and one-dimensional cases. The results show that the degree of recognition with spatial restriction is increased beyond that of the classical example, provided the input bias favors signal over noise $\left(X_{1}>X_{2}\right)$. If the input is unbiased, then the degree of recognition is the same as in the classical example, regardless of spatial restriction. If the input bias favors noise over signal, then the degree of recognition is actually decreased below that seen in the classical example. We will come back to a discussion of this behavior in the last section. 


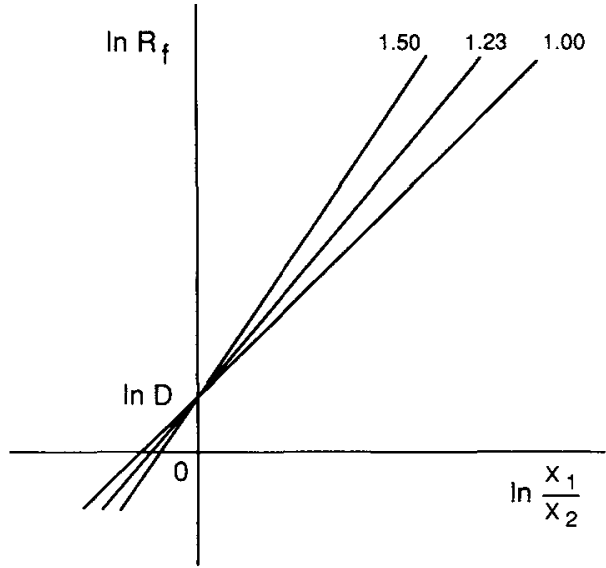

Figure 7. Degree of recognition as a function of the input bias for a fractal recognition process. The three functions represent fractal recognition constrained by various degrees of spatial restriction. The numbers represent the kinetic order $(g)$ associated with the fractal dimension; these are characteristic of processes operating within a three-dimensional space $(1.00)$, a two-dimensional surface (1.23), and a one-dimensional channel (1.50). The slope is equal to the kinetic order $g$, and the axes and vertical intercept are as described in Fig. 6 . See text for further discussion.

The fractal recognition function also can be written as:

$$
\left(\frac{R_{\mathrm{f}}}{R}\right)=\left(\frac{X_{1}}{X_{2}}\right)^{g-1}
$$

where $R$ is the recognition function defined for the classical example. A plot of this equation for different values of $g$ is shown in Fig. 8. This method of viewing the recognition function illustrates the fractal contribution relative to the classical contribution. When $g=1$ the $R_{\mathrm{f}} / R$ ratio is one, which corresponds to the horizontal axis. The distance above the horizontal, in the cases with spatial restriction, indicates the fractal kinetic enhancement in recognition. Clearly, there is no enhancement when the input is unbiased $\left(X_{1}=X_{2}\right)$, and enhancement increases with the degree of bias favoring the input signal.

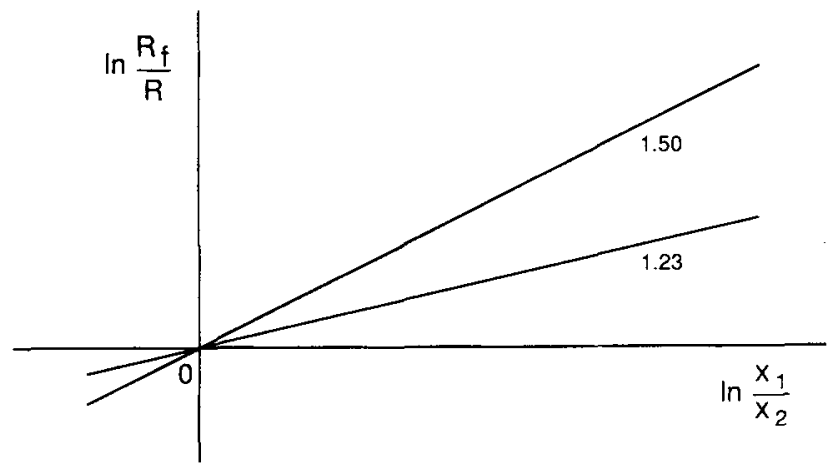

Figure 8. Fractal recognition relative to classical recognition as a function of input bias. This is an alternative view of the recognition function showing that the degree of recognition increases with the degree of spatial restriction and input bias. The logarithm of the fractal recognition function $R_{\mathrm{f}}$ divided by the classical recognition function $R$ is represented on the vertical axis, and the logarithm of the input $\mathrm{S} / \mathrm{N}$ ratio (bias) is represented on the horizontal axis. The numbers represent the kinetic order $g$, as in Fig. 7 ; the slope in this case, however, is equal to $g-1$.

\section{Recognition by enzyme kinetic discrimination}

The behavior of the enzyme kinetic mechanism in Fig. 5 also becomes altered when it is imbedded in a twodimensional membrane or a one-dimensional channel. The output in this case is taken to be $v_{5}$, the flux of the proper product $X_{5}$, and $v_{6}$, the flux of the analog product $X_{6}$.

The transformation now is represented by the following equations in the Power-Law formalism:

$$
\begin{gathered}
\mathrm{d} X_{3} / \mathrm{d} t=\alpha_{3} X_{1}^{g_{31}} X_{7}^{g_{37}}-\left(\alpha_{5}+\beta_{3}\right) X_{3} \\
\mathrm{~d} X_{4} / \mathrm{d} t=\alpha_{4} X_{2}^{8_{42}} X_{7}^{8_{47}}-\left(\alpha_{6}+\beta_{4}\right) X_{4} \\
X_{7}=X_{\mathrm{T}}-X_{3}-X_{4}
\end{gathered}
$$

At steady state $\left(\mathrm{d} X_{i} / \mathrm{d} t=0\right)$ with negligible product concentration:

$$
\begin{aligned}
& X_{3}=\frac{\alpha_{3}}{\alpha_{5}+\beta_{3}} X_{1}^{g_{11}} X_{7}^{8_{37}} \\
& X_{4}=\frac{\alpha_{4}}{\alpha_{6}+\beta_{4}} X_{2}^{8_{42}} X_{7}^{g_{47}}
\end{aligned}
$$

and the degree of fractal recognition, denoted by the symbol $R_{\mathrm{f}}$, is then:

$$
R_{\mathrm{f}}=\frac{v_{5}}{v_{6}}=\frac{\alpha_{5} X_{3}}{\alpha_{6} X_{4}}=\left[\frac{\alpha_{3} \alpha_{5}\left(\alpha_{6}+\beta_{4}\right)}{\alpha_{4} \alpha_{6}\left(\alpha_{5}+\beta_{3}\right)}\right] \frac{X_{1}^{g_{31}} X_{7}^{g_{37}}}{X_{2}^{g_{4}} X_{7}^{g_{47}}}
$$

It is clear that this recognition function is much more complex than that of the classical example [Eqn. (17)]. Nevertheless, one can determine the fractal recognition function numerically for any combination of values for the kinetic orders, rate constants, and total concentration of enzyme, although this will not be presented here.

Again, for the case in which the kinetic orders are all equal and have values for the heterodimeric associations that are one half those for the analogous homodimeric associations, we can define $g_{31}=g_{42}=g_{37}=g_{47}=g$. The $X_{7}$ terms in the fractal recognition function then cancel, and the simplified function becomes:

$$
R_{\mathrm{f}}=\left[\frac{\alpha_{3} \alpha_{5}\left(\alpha_{6}+\beta_{4}\right)}{\alpha_{4} \alpha_{6}\left(\alpha_{5}+\beta_{3}\right)}\right]\left(\frac{X_{1}}{X_{2}}\right)^{g}
$$

or

$$
R_{\mathrm{f}}=D\left(\frac{X_{1}}{X_{2}}\right)^{g}
$$

where $D$ is the overall discrimination factor as defined for the classical example. It should be noted that, as in the previous example, the value of $D$ will undoubtedly change with spatial restriction, although this thermodynamic effect will not concern us here. Again, the fractal recognition function also can be written as:

$$
\left(\frac{R_{\mathrm{f}}}{R}\right)=\left(\frac{X_{1}}{X_{2}}\right)^{g-1}
$$

where $R$ is the recognition function defined for the classical example.

Since the form of these last two equations is exactly the same as it was in the previous example, the plots in Figs 7 and 8 apply here as well. Thus, there is no 
enhancement when the input is unbiased $\left(X_{1}=X_{2}\right)$, and enhancement increases with the degree of bias favoring the input signal.

\section{DISCUSSION}

Classical recognition, as defined here, depends upon both the inherent discrimination of the molecular mechanism and the input bias of the environment [e.g., see Eqn. (1)]. A large discrimination factor allows a small input signal to be recognized clearly even in the presence of abundant noise. For example, the amino acid tyrosine is 50 times less abundant than glutamate in Saccharomyces cerevisiae (Holden, 1962), and yet tyrosyl-tRNA synthetase $(D>5000)$ achieves a degree of recognition greater than 100 (Igloi et al., 1978), or 10000 if one can generalize the results of Loftfield (Loftfield, 1963; Loftfield and Vanderjagt, 1972). Such large discrimination factors are readily obtained even for molecules that exhibit relatively modest differences in structure. The role of input bias is less important under these circumstances.

The role of input bias becomes more important when molecules are so similar in structure that there is little basis for discrimination. In the extreme, a mechanism with no inherent discrimination $(D=1)$ could achieve a degree of recognition equal to 100 (1\% error) only if the signal was 100 times more abundant than the noise in the input environment. While such a bias might fortuitously favor one molecule over an alternative in the intracellular environment, the symmetrical problem of recognizing the alternative in such an environment would then become impossible.

This problem still can be important when there is a low but significant level of discrimination. For example, a binding site designed to accommodate isoleucine will not be able to reject completely the analog valine, which is structurally similar but slightly smaller because it lacks a methyl group. Pauling (1957) calculated, and others (Baldwin and Berg, 1966; Loftfield and Eigner, 1966) measured, a discrimination factor of $<100$ for this task, yet the overall degree of recognition for isoleucine in the cell approaches 10000 (Loftfield, 1963; Loftfield and Vanderjagt, 1972). If this degree of overall recognition were to be achieved with a discrimination factor of 100 and an input bias of 100 in favor of isoleucine (which is decidedly not the case), then the corresponding degree of recognition for valine by its cognate enzymes would require a discrimination factor of 1000000 . However, the discrimination factor for valine in the presence of isoleucine is about 100000 (Fersht, 1979), which would be an order of magnitude too small, so there must be another solution to the problem of low inherent discrimination.

Two completely different strategies have evolved to deal with the problem of low inherent discrimination: composite mechanisms for discrimination [e.g., see Eqn. (2)] and control mechanisms for regulating the concentrations of intracellular metabolites. An example of a composite mechanism is provided by isoleucyltRNA synthetase. In addition to aminoacylation of tRNA molecules, this enzyme also preferentially hydrolyzes incorrect aminoacyl-tRNA molecules that involve valine. Fersht (1979) describes this in terms of a double-sieve mechanism: the first screen is coarse enough to allow the larger isoleucine molecules through as well as the smaller valine molecules, the second screen is fine enough to select only the smaller valine molecules for rejection. This combination of individual discrimination factors is able to achieve the required overall discrimination factor of 10000 . There is an energy cost to the accuracy achieved, because the proofreading function occurs at the expense of a highenergy bond. Indeed, there is a well-defined costaccuracy relationship independent of the molecular details of the mechanism (Savageau and Freter, 1979; Freter and Savageau, 1980). This cost of proofreading is in addition to the free energy consumed in driving the overall reaction far from thermodynamic equilibrium, which is an energy cost whether or not kinetic proof reading takes place.

The second strategy, evolution of control mechanisms, has led to a number of ways for regulating the levels of important metabolites in the cell. In the case of amino acids, these mechanisms include end-product inhibition of biosynthetic enzymes, repression of genes encoding biosynthetic enzymes, and induction of genes encoding catabolic enzymes. Such mechanisms perform a number of important functions in the cell and have been the subject of considerable analysis (e.g., see Savageau, 1976; Voit, 1991). In each case, an elevation in the concentration of an amino acid leads to a compensatory response tending to return the concentration to its predisturbance level. Intracellular control mechanisms such as these are ubiquitous, even among multicellular organisms whose individual cells experience a relatively constant environment by virtue of their participation in a communal homeostasis. One sees a particularly luxuriant flowering of molecular control mechanisms among free-living cells that experience the greatest extremes of environmental variation. As a consequence of these rich networks of regulatory mechanisms within cells, the input bias for any particular molecular recognition process is buffered against radical change.

The implications of fractal kinetics for molecular recognition go beyond those discussed above in the context of classical kinetics. The principal distinction lies in the ability of fractal kinetics to nonlinearly transform, rather than proportionally transmit, the input bias [e.g., see Eqn. (37)]. The transformation by a power-law function allows for disproportionate enhancement of recognition when the input bias is greater than one. For example, with a kinetic order $g=1.5$, an input bias of 100 is transformed into a factor of 1000 in the fractal recognition function. Thus, the degree of recognition is amplified 10 -fold over what it would otherwise be with classical kinetics.

The power-law transformation of the input bias also allows for a sharpening of threshold in the recognition function, since recognition is disproportionately diminished when the input bias is less than one. For example, an input bias of $1 / 100$ is transformed into a factor of $1 / 1000$, and the degree of recognition is attenuated 10 -fold from what it would otherwise be with classical kinetics. Thresholds of this sort are common in the intracellular processing of biochemical signals.

Dimerization of cell surface receptors is emerging as a common design feature of signal transduction pathways in nearly all organisms (e.g., see Brugge, 1993; 
and Marx, 1993). Bimolecular association with spatial restriction is just the condition under which one might expect to find fractal recognition playing an important role. There also is kinetic evidence for a very high rate of these dimeric associations (Wang and Smith, 1987; Fay et al., 1991; Felder et al., 1993), which further suggests that diffusional limitation and fractal kinetics may play an important role in vivo. As Felder et al. (1993) observed in vitro, the 'on' rate cannot be measured directly because of diffusion problems, so they calculated it indirectly from measurements of the 'off' rate and the concentration equilibrium constant. This method of indirect determination must be carefully evaluated because the concentration equilibrium constant may be different in vitro and in vivo due to thermodynamic (Minton, 1992) and kinetic (Savageau, unpublished data) effects of spatial restriction. It is tempting to speculate on the amplification and threshold behavior that might be achieved with fractal recognition at the earliest stage (and possibly subsequent stages) of these cascade systems.

Similar considerations may apply to cell-cell interactions (Foote and Milstein, 1991; Lawrence and Springer, 1991), although the association of two surfaces with potentially many individual receptor molecules is obviously much more complex than a simple bimolecular reaction.

Let us briefly re-examine the scope of this paper and consider some of the implied limitations before drawing to a conclusion. The primary focus has been the implications of classical and fractal kinetics for molecular recognition. Note that classical recognition [e.g., Eqns. (10) and (17)] is a linear function of the ratio of ligand concentrations. This is often supported by experimental data obtained in vitro under conditions that approach that of ideal solution chemistry. The conditions in a living cell are undoubtedly quite different (Clegg, 1984; Srere et al., 1989; Minton, 1992; Savageau, 1992). Under these nonideal conditions the recognition function need not be a linear function, or even a single-valued function, of the ratio of concentrations. As we have seen, the more general cases of fractal recognition [e.g., Eqns. (27) or (36)] are neither linear nor single-valued functions of the ratio of concentrations. The special cases of fractal recognition examined in this paper [e.g., Eqns (28) and (37)] are nonlinear but still single-valued functions of the ratio; only in this latter respect are they similar to classical recognition.

Although this paper has emphasized the implications of fractal kinetics in molecular recognition processes, the scope is actually more general. In fact, we have been examining the implications of power-law kinetics, which not only represent the kinetics of fractal phenomena, but also the kinetics of other nonideal phenomena. Power-law kinetics follow logically from fractal phenomena, but the converse is not necessarily true; the observation of power-law kinetics does not imply fractal phenomena.

Essentially any nonlinear function of biological interest that relates the rate of a reaction to the variables that influence the reaction can be represented accurately by a Taylor series expansion. Indeed, one often can represent the behavior accurately with just the first few terms of such an expansion, provided the range of variation about the nominal values for the variables is not too great. The local power-law representation that has been used in this paper derives from just such a truncated Taylor series. However, the series is developed in a logarithmic coordinate system rather than the usual Cartesian coordinate system; this yields an accurate representation over a wider range of operation and gives rise to power laws when the equations are transformed back into the Cartesian coordinate system (Savageau, 1972).

If a nonideal process is pushed to sufficient lengths, usually beyond the normal physiological range (Sorribas and Savageau, 1989), the nonlinearity exhibited may be so great that the local power-law representation will no longer provide an accurate representation. In such cases, one must resort to more general forms of nonlinear analysis, such as nonlinear recasting within the Power-Law formalism (Savageau, 1993).

In conclusion, the results in this paper suggest that recognition in the presence of a favorable input ratio would take advantage of the amplification provided by fractal kinetics. This in turn implies an emphasis on rapid reactions, because fractal kinetics arise under conditions of diffusional limitation. On the other hand, recognition in the presence of an unfavorable input ratio would not be expected to employ fractal kinetics, but instead to emphasize slow reactions. Thus, fractal kinetics implies an intimate relationship between design of the physiological mechanisms regulating the environmental bias being presented to a process and design of the molecular process itself to achieve a given degree of discrimination.

\section{Acknowledgements}

This work was supported in part by United States Public Health Service Grant GM-30054 from the National Institutes of Health. I thank Drs $R$. Jove and $Y$. Shimizu for suggestions regarding kinetic data relevant to signal transduction and cellular interactions, and Dr A. Minton for thoughtful comments that helped to clarify the presentation.

\section{REFERENCES}

Baldwin, A. N., and Berg, P. (1966). Transfer ribonucleic acidinduced hydrolysis of valyladenylate bound to isoleucyl ribonucleic acid synthetase. J. Biol. Chem. 241, 839-845.

Brugge, J. S. (1993). New intracellular targets for therapeutic drug design. Science 260, 918-919.

Clegg, J. S. (1984). Properties and metabolism of the aqueous cytoplasm and its boundaries. Am. J. Physiol. 246, R133-R151.

Erdi, P., and Toth, J. (1989). Mathematical Models of Chemical Reactions. Princeton University Press, Princeton.
Fay, S. P., Rosner, R. G., Swann, W. N., and Sklar, L. A. (1991) Real-time analysis of ligand, receptor, and $G$ protein by quantitative fluorescence flow cytometry. Biochemistry 30 , 5066-5075.

Felder, S., Zhou, M., Hu, P., Urena, J., Ulirich, A., Chaudhuri, M., White, M., Shoelson, S. E., and Schlessinger, J. (1993). SH2 domains exhibit high-affinity binding to tyrosinephosphorylated peptides yet also exhibit rapid dissociation and exchange. Mol. Cell. Biol. 13, 1449-1455.

Fersht, A. (1979). Editing mechanisms in the aminoacylation of 
tRNA. In Transfer RNA, ed. by P. Schimmel, D. Soll and J. Abelson, pp. 247-254. Cold Spring Harbor Laboratory Press, Cold Spring Harbor.

Fersht, A. (1985). Enzyme Structure and Mechanism, 2nd edn. W.H. Freeman and Company, San Francisco.

Foote, J., and Milstein, C. (1991). Kinetic maturation of an immune response. Nature 352, 530-532.

Freter, R. R., and Savageau, M. A. (1980). Proofreading systems of multiple stages for improved accuracy of biological discrimination. J. Theoret. Biol. 85, 99-123.

Galfi, L. and Racz, Z. (1988), Properties of the reaction front in an $A+B \rightarrow C$ type reaction-diffusion process. Phys. Rev. A 38, $3151-3154$.

Holden, J. T. (1962). The composition of microbial amino acid pools. In Amino Acid Pools, ed. by J. T. Holden, pp. 73-108. Elsevier, Amsterdam.

Igloi, G. L., von der Haar, F. and Cramer, F. (1978). Aminoacyl-tRNA synthetases from yeast: Generality of chemical proofreading in the prevention of misaminoacylation of tRNA. Biochem. 17, 3459-3468.

Jiang, Z., and Ebner, C. (1990). Simulation study of reaction fronts. Phys. Rev. A 42, 7483-7486.

Koo, Y.-E. L., and Kopelman, R. (1991). Space- and time-resolved diffusion-limited binary reaction kinetics in capillaries: experimental observation of segregation, anomalous exponents, and depletion zone. J. Stat. Phys. 65, 893-918.

Kopelman, R. (1986). Rate processes on fractals: theory, simulations, and experiments. J. Stat. Phys, 42, 185-200.

Lawrence, M. B., and Springer, T. A. (1991). Leukocytes roll on a selectin at physiologic flow rates: distinction from and prerequisite for adhesion through integrins. Cell 65, 859873.

Loftfield, R. B. (1963). The frequency of errors in protein biosynthesis. Biochem. J. 89, 82-92.

Loftfield, R. B., and Eigner, E. A. (1966). The specificity of enzymic reactions. Aminoacyl-soluble RNA ligases. Biochim. Biophys. Acta 130, 426-448.

Loftfield, R. B. and Vanderjagt, D. (1972). The frequency of errors in protein biosynthesis. Biochem. J. 128, 1353-1356.
Marx, J. (1993). Forging a path to the nucleus. Science 260. $1588-1590$.

Minton, A. P., (1992). Confinement as a determinant of macromolecular structure and reactivity. Biophys. J. 63, 1090-110.

Newhouse, J. S. and Kopelman, R. (1988). Steady-state chemical kinetics on surface clusters and islands: segregation of reactants. J. Phys. Chem. 92, 1538-1541.

Pauling, L. (1957). The probability of errors in the process of synthesis of protein molecules. In Festschr. Arthur Stoll, pp. 597-602. Birkhauser, Basel.

Savageau, M. A. (1972). The behavior of intact biochemical control systems. Curr. Top. Cell. Regul. 6, 63-130.

Savageau, M. A. (1976). Biochemical Systems Analysis: a Study of Function and Design in Molecular Biology. Addison-Wesley, Reading, MA.

Savageau, M. A. (1992). A critique of the enzymologist's test tube. In Fundamentals of Medical Cell Biology, ed. by E. E. Bittar, Vol. 3A, pp. 45-108. JAl Press Inc., Greenwich, CT.

Savageau, M. A. (1994). Power-law formalism: a canonical nonlinear approach to modeling and analysis. In Proceedings of the First World Congress of Nonlinear Analysts, in press. Walter de Gruyter, Berlin.

Savageau, M. A., and Freter, R. R. (1979). Energy cost of proof reading to increase fidelity of transfer ribonucleic acid aminoacylation. Biochemistry 18, 3486-3493.

Sorribas, A., and Savageau, M. A. (1989). A comparison of variant theories of intact biochemical systems l: enzymeenzyme interactions and biochemical systems theory. Math. Biosci. 94, 161-193.

Srere, P., Jones, M. E., and Matthews, C. (1989). Structural and Organizational Aspects of Metabolic Regulation. Alan R. Liss, New York.

Voit, E. O. (1991). Canonical Nonlinear Modeling: S-System Approach to Understanding Complexity. Van Nostrand Reinhold, New York.

Wang, H. M., and Smith, K. A. (1987). The interleukin 2 receptor: functional consequence of its biomolecular structure. $J$ Exp. Med. 166, 1055-1065. 